\title{
Feasibility and Preliminary Efficacy Data from a Computerized Cognitive Intervention in Children with Chromosome 22q11.2 Deletion Syndrome
}

\author{
Waverly Harrell ${ }^{1}$, Shaun Eack ${ }^{2}$, Stephen R. Hooper ${ }^{3}$, Matcheri S. Keshavan ${ }^{4}$, Melanie S. \\ Bonner $^{5}$, Kelly Schoch ${ }^{1}$, and Vandana Shashi ${ }^{1}$ \\ ${ }^{1}$ Division of Medical Genetics, Department of Pediatrics, Duke University Medical Center, Durham \\ NC \\ ${ }^{2}$ Department of Social Work, University of Pittsburgh, Pittsburgh, PA \\ ${ }^{3}$ Department of Psychiatry and the Carolina Institute for Developmental Disabilities, University of \\ North Carolina School of Medicine, Chapel Hill NC \\ ${ }^{4}$ Department of Psychiatry, Beth Israel Deaconess Medical Center, Boston MA \\ ${ }^{5}$ Department of Psychology, Duke University Medical Center, Durham NC
}

\subsection{Introduction}

Chromosome 22q11.2 deletion syndrome (22q11DS), also called velocardiofacial syndrome or DiGeorge syndrome, results from a microdeletion on the long arm of chromosome 22 at band 11.2 (Driscoll, Budarf, \& Emanuel, 1992; Shprintzen, 2008). The phenotypic presentation is highly variable, with many children with $22 q$ experiencing multiple medical problems (Philip \& Bassett, 2011; Shprintzen, 2008). Psychiatric disorders occur in about $60 \%$ of the children, including anxiety and depressive disorders and attention deficit hyperactivity disorder (ADHD) (Jolin, Weller, \& Weller, 2009; Papolos et al., 1996; Young, Shashi, Schoch, Kwapil, \& Hooper, 2011). As they reach adolescence, a subset of children with 22q11DS begin to show symptoms of psychosis or experience transient psychotic episodes (Baker \& Skuse, 2005; Debbane, Glaser, David, Feinstein, \& Eliez, 2006; Stoddard, Niendam, Hendren, Carter, \& Simon, 2010). Approximately 25-40\% develop schizophrenia, another psychotic disorder, bipolar disorder, or major depression in adolescence or adulthood (Murphy, Jones, \& Owen, 1999; Papolos et al., 1996; Pulver et al., 1994; Shprintzen, Goldberg, Golding-Kushner, \& Marion, 1992).

Almost all children with 22q11DS show some level of cognitive impairment. IQ is reported to range from the 40s to the low 100s, with the average in the 70s (De Smedt et al., 2007; Lewandowski, Shashi, Berry, \& Kwapil, 2007; Moss et al., 1999; Woodin et al., 2001). Deficits in processing speed and aspects of executive function, including attention and working memory, impair academic performance (Debbane, Glaser, \& Eliez, 2008; Niklasson, Rasmussen, Oskarsdottir, \& Gillberg, 2005). In our cohort of 85 children with

(C) 2013 Elsevier Ltd. All rights reserved

Corresponding Author: Vandana Shashi, MD Associate Professor of Pediatrics Division of Medical Genetics Duke University Medical Center Box 103857 Durham NC 27710 Phone: (919) 681-2616 Fax: (919) 668-0414 vandana.shashi@ duke.edu.

Publisher's Disclaimer: This is a PDF file of an unedited manuscript that has been accepted for publication. As a service to our customers we are providing this early version of the manuscript. The manuscript will undergo copyediting, typesetting, and review of the resulting proof before it is published in its final citable form. Please note that during the production process errors may be discovered which could affect the content, and all legal disclaimers that apply to the journal pertain. 
22q11DS, nearly two-thirds scored in the lowest quartile in both reading and mathematics, and low achievement was associated with lower global assessment of function (GAF) scores (Curtiss, Hooper, Schoch, \& Shashi, 2013). No evidence-based pharmacological or nonpharmacological interventions have been developed to improve cognitive deficits in individuals with 22q11DS. Because children with 22q11DS are at risk for developing schizophrenia, and the pattern of cognitive impairments observed in children with 22q11DS is consistent with those associated with schizophrenia (Lewandowski et al., 2007), interventions with demonstrated efficacy in individuals with schizophrenia would be expected to be effective in children with 22q11DS.

Cognitive interventions have been developed to improve cognitive, social, and functional deficits for adults with schizophrenia (Eack, Hogarty, Greenwald, Hogarty, \& Keshavan, 2011). Computerized software programs developed by Posit Science, Inc. (San Francisco, CA) have been shown to improve memory and attention in adults with stabilized schizophrenia (Mahncke et al., 2006a, Fisher et al., 2010, Genevsky et al., 2010), with more intensive interventions being associated with broader cognitive improvements (Mahncke et al., 2006b, Smith et al., 2009, Murthy et al., 2012). Exercises presented auditory stimuli, including tones, syllables, verbal instructions, and a narrative, initially slowly with exaggerated articulation, then gradually more quickly and subtly until they resembled realistic rapid speech. Difficulty increased gradually so that participants were always correct on approximately $85 \%$ of their answers, and correct answers were rewarded with visual stimuli and points (Smith et al., 2009). Such computerized cognitive remediation programs are safer and less costly than pharmacologic treatments, may improve cognitive impairments more directly (Keefe and Harvey, 2012), and could hold promise for individuals with 22q11DS. A recent meta-analysis of 40 studies of cognitive remediation for adults with schizophrenia found that these programs produced a small to moderate durable effect on cognitive functioning, including significant improvements in attention, processing speed, verbal working memory, verbal learning, problem-solving, and social cognition (Wykes, Huddy, Cellard, McGurk, \& Czobor, 2011).

These programs, including the Posit Science modules, are based on the concept of neuroplasticity, the ability of the brain to remodel neural pathways in response to repeated external stimuli and thereby alter functionality (Mahncke, Bronstone, \& Merzenich, 2006). Both functional and structural brain changes have been reported in association with computerized training. In adults with schizophrenia, computerized training targeting auditory processing and social cognition has been shown to increase functional activation of brain areas supporting social cognition (Hooker et al., 2012), and computerized cognitive training followed by Cognitive Enhancement Therapy has been associated with preservation of gray matter in medial temporal brain areas, with gray matter changes mediating treatment effects (Eack et al., 2010).

Computerized cognitive interventions have also resulted in improvements in children with ADHD and children with cognitive impairments, both populations with features common to many children with 22q11DS. Computerized cognitive training programs targeting attention and working memory have been employed to treat ADHD in children, and working memory gains have been found to last for up to 6 months (Holmes et al., 2010). A recent metaanalysis found that such programs did produce significant improvements, but only when endpoints included the ratings of parents or teachers who knew which children received the intervention (Sonuga-Barke et al., 2013). Computerized training has been shown to improve working memory in adolescents born at extremely low weights who had average IQ $<80$ (Lohaugen et al., 2011). A computerized intervention targeting specific mathematical skills has been shown to improve those skills in children with idiopathic developmental disability (mean IQ 66) and in a subset of children with Fragile X syndrome and comparable IQs 
(Hammond, Hirt, \& Hall, 2012). One recent study examining the effects of 6 to 8 hours of computer training targeting non-verbal reasoning and working memory in children with IQs $<70$ found that while the training did not have significant effects for the whole intervention group as compared with controls, participants who made greater progress during training were more likely to show transfer of skills directly after training. Girls with higher baseline verbal working memory and no additional diagnoses were more likely to benefit from the intervention. However, benefits were not sustained at one year, and the authors speculated that more extended training might be necessary to maintain benefits (Soderqvist, Nutley, Ottersen, Grill, \& Klingberg, 2012).

With this background, we aimed to develop a cognitive remediation program for children with 22q11DS. This paper provides data from the first intervention study of its kind. Our hypothesis was that a computerized cognitive remediation grounded in neuroplasticity research, with exercises designed to improve attention, working memory, and processing speed, would improve cognition in adolescents with 22q11DS. We designed a 12-week intervention utilizing the computerized cognitive remediation program (CCRP) developed by Posit Science. We present feasibility data and preliminary pre- and post-cognitive intervention findings providing evidence of the validity of this novel approach.

\subsection{Methods}

\subsubsection{Demographics}

Participants were recruited through the Duke University Medical Center genetics clinic and the North Carolina support group for families of children with 22q11DS. The participants included 23 children with 22q11DS: 13 children in the intervention group and 10 nonrandomly assigned age- (within 9 months) and gender-matched children in the control group. All participants had diagnoses of 22q11DS confirmed by fluorescence in-situ hybridization. Participants with an estimated full scale IQ (FSIQ) $<55$ were excluded, since they would be expected to have difficulty completing the required tasks. Participants with active psychosis were excluded because psychosis would be expected to affect the cognitive assessment results and compliance with the study.

There were no significant differences between the intervention and control groups in age, gender, or race (see Table 1). Socioeconomic status (SES), which has been found to influence psychological function in children with 22q11DS (Shashi et al., 2010), was also similar in both groups, as was Global Assessment of Functioning (GAF). There were no significant group differences in medication status for ADHD (3/10 in control group and 2/13 in the intervention group were on ADHD medications, FET $p=.63$ ). Four of the 13 participants in the intervention group were on psychotropic medications in comparison to $1 / 10$ in the control group, FET $\mathrm{p}=.63$ (selective serotonin reuptake inhibitors in all cases, except for one control subject taking a tricyclic antidepressant for anxiety). Although the intervention did not target psychiatric manifestations of 22q11DS, we assessed the psychiatric status of both groups at baseline (T1) to see whether significant differences would need to be accounted for in the statistical analyses and found no differences in psychiatric diagnoses among groups. Four of 13 and 3/10 in the intervention and control groups met criteria for an anxiety disorder and $3 / 13$ and 3/10 for ADHD, respectively (FET $\mathrm{p}=.66$ ), using the Computerized Diagnostic Interview Schedule for Children (NIMHCDISC, 2004).

\subsubsection{Cognitive Outcome Measures}

Psychological assessments that would provide outcome measures were administered to participants at T1 and following the CCRP program, or, for the control group, 12 weeks 
from baseline (T2). Assessments were conducted by a trained postdoctoral fellow or research assistant under supervision of a licensed psychologist. To prevent bias, the assessor was not involved in administration or supervision of the CCRP and was blinded to group assignment. All 10 control participants and 10/13 intervention participants were reassessed after the 12-week intervention period. Parents reported no changes in SES or participants' medications over the course of the study. Reassessments all occurred within 4 weeks after the intervention ended.

The computerized cognitive intervention focused on improving attention, working memory, and processing speed. Consequently, we focused on three primary domains for our cognitive outcomes: Attention, Memory, and Executive Function. In addition, we combined all of the measures to yield an overall cognitive composite score as an additional outcome measure for our feasibility study. The psychological assessments chosen to ascertain functioning in the above domains were as follows: 1) Attention: Continuous Performance Task, IP version (Cornblatt, Risch, Faris, Friedman, \& Erlenmeyer-Kimling, 1988), and Child Behavior Checklist (CBCL) Attention T-score (Biederman et al., 1993); 2) Memory: California Verbal Learning Test (CVLT) Children's version (Delis, 1993); and 3) Executive Function: Digit Span subtest of the Wechsler Intelligence Scale for Children (WISC) $4^{\text {th }}$ edition or Wechsler Adult Intelligence Scale (WAIS) $4^{\text {th }}$ edition, depending on participant's age (Wechsler, 2003, 2008), Spatial Span subtest on the WISC-IV Integrated (McCloskey \& Maerlender, 2005), Stroop Color-Word Test (Stroop, 1935), and Porteus Mazes-Vineland revision (Krikorian \& Bartok, 1998).

\subsubsection{CCRP}

The intervention consisted of a computerized program (BrainWorks ${ }^{\mathrm{TM}}$, Posit Science, CA) completed at home. Participants were asked to play three games per session four times a week for 12 weeks, totaling approximately 32 hours of intervention. This length of time was chosen based on the number of hours of computerized intervention shown to improve memory and attention in adults with schizophrenia (Smith et al., 2009). Control participants were not asked to complete computer activities. Prior to initiation of the study, the study team conferred with the scientific team at Posit Science to choose from among existing BrainWorks ${ }^{\mathrm{TM}}$ exercises the ones that would most appeal to the study participants (children with cognitive impairments) and that would target the domains in which deficits are most common in individuals with 22q11DS. Since visual-spatial processing deficits are almost universal in individuals with this condition, we opted to include two visual exercises and one auditory exercise in each session.

The exercises comprised 8 games. Two were visual. One, Pet Wrangler, required the participant to track multiple objects moving across a screen. This game was intended to improve attention and visual working memory. The other visual game, Speed Trap, required remembering the location of briefly presented pictures moving increasingly far from a central point. This exercise targeted processing speed and attention. Six of the games required the participant to remember sounds: tones, phonemes, syllables, a narrative, or instructions. All of these games were intended to improve verbal working memory. The games Test Driver and Navigator also targeted processing speed, presenting stimuli for increasingly brief intervals or presenting increasingly similar stimuli, respectively. The games Coffee Break, Stage Crew, Brain Blog, and Audio Mash-Up targeted attention, presenting increasingly lengthy sequences of auditory stimuli. The program was piloted with four children with 22q11DS who had full-scale IQ scores ranging from 56-88. All four were able to play the exercises with at least $60 \%$ accuracy, with a mean of 3 exercises per session.

After completion of the initial psychological testing, participants began the CCRP. Each participant was monitored by the study personnel as he or she first logged into the program 
and played one exercise (approximately 13 minutes) to ensure that he or she could play the exercises without parental assistance at home. After completing the program, each participant again played one game in front of study personnel to show that he or she was capable of playing the games independently. During each session at home the children played three exercises, each lasting approximately 13 minutes. The exercises were adaptive, first establishing the level at which $85 \%$ of a participant's responses were correct and then becoming more difficult as performance improved. A participant initiated each trial at his or her own pace, ensuring that the participant's attention was engaged at the beginning of the trial. Virtual rewards were built into the program, including badges and "brain bucks" that could be used to buy items for a virtual house pictured on the computer. The investigators also sent encouraging messages to the participants through the program and contacted parents weekly with updates on their children's progress. Participants were paid $\$ 10$ for each complete week of work.

\subsubsection{Statistical Analyses}

A series of intent-to-treat analyses using linear mixed-effects models was conducted with all 23 participants enrolled in the study, regardless of attrition, to examine the efficacy of CCRP on proximal cognitive outcomes in 22q11DS. To protect against the inflation of Type I error and increase statistical power, primary analyses were conducted on an overall composite index of cognitive function. This index was computed by scaling individual cognitive test scores to a common $(z)$ metric based on baseline (pre-treatment) scores, reverse scoring relevant tests such that higher values would reflect better cognitive function (i.e., Stroop Times 1 and 2, CBCL Attention), and averaging across these tests. The resulting multivariate cognitive composite proved to have sufficient internal consistency $(a=.74)$. After conducting primary analyses examining the effects of CCRP on overall cognitive function, secondary analyses explored the differential effects of the intervention on univariate components of this composite. All analyses made use of random intercept mixedeffects models and adjusted for potential age and gender effects on cognitive outcomes. In addition, because the control group demonstrated some greater pre-treatment function on two of the cognitive tests (CPT: $\mathrm{t}=2.44, \mathrm{p}<.05$; Porteus Mazes: $\mathrm{t}=2.03, \mathrm{p}=.05$ ), all analyses also accounted for between-group differences in initial status in the cognitive outcome being investigated. Missing data (in 3 of the 13 CCRP patients who terminated treatment early) were handled during maximum-likelihood estimation using the expectationmaximization approach (Dempster AP, 1977) at the time of analysis, which is the preferred approach to handling missing treatment data (Hamer \& Simpson, 2009). To provide an estimate of the magnitude of the effects of CCRP on cognition, within- and between-group Cohen's $d$ effect sizes were computed based on model-adjusted means and pre-treatment standard deviations pooled across both the intervention and control groups.

\subsection{Results}

\subsubsection{Feasibility}

Of the 13 participants who began the computerized cognitive intervention, nine (69\%) completed the program. One of the four other children completed more than half the assigned exercises, while the other three (23\%) completed fewer than half. Barriers to implementing the CCRP in these four children were identified as inconsistent or unreliable internet access in two cases and lack of motivation on the part of parents or children in the other two cases. The specific barriers to computer access were that one child's family sold their laptop and the other child's custodial parent blocked internet access due to concerns about the child's online social networking. Neither family was responsive to the researchers' offering to provide computer access. Parents and children in the intervention group were 
surveyed at six and 12 weeks of the program to gauge acceptability and satisfaction with the program. The results are presented in Tables 2 and 3.

\subsubsection{Adherence to the Program}

Adherence was automatically tracked by the computer program and monitored weekly by the study staff. The nine participants who completed the intervention and the $10^{\text {th }}$ who finished more than half the exercises were included in the analyses for adherence. The mean number of hours spent on the CCRP for these participants was $29.7 \pm 3.5$, and the mean number of exercises completed was $131 \pm 18.5$.

\subsubsection{Preliminary Cognitive Outcomes}

At the end of the intervention period, 10 control participants and 10 intervention participants (nine who completed and the $10^{\text {th }}$ subject who completed the majority of the program) returned for psychological reassessments within four weeks of completion of the program. Statistical tests of between-group differences from mixed-effects models produced only two significant differences. These were noted for differential improvements in the Cognitive Composite, reflecting an overall positive effect for the treatment group (Table 4) and in Stroop Time 1, reflecting an increase in simple processing speed for the treatment group. In addition, differences in magnitude of the effects between groups were striking, with the Treatment Group showing significant differential improvements on nearly every cognitive task. This was particularly evident on the Cognitive Composite, for which the treatment group demonstrated a medium to large advantage over the control group (Cohen's $d=.79$ ). When the change in GAF score from $\mathrm{T} 1$ to $\mathrm{T} 2$ between the two groups was observed, the intervention group maintained their GAF scores, compared to a decline in the control group. The difference was not significant but had a medium effect size $(\mathrm{t}=.953, \mathrm{p}=\mathrm{ns}$, Cohen's $d$ $=0.5$ ).

\subsection{Discussion}

Since 22q11DS is a common genetic condition, second only to Down syndrome among autosomal chromosomal disorders, the public health impact from the psychological morbidity associated with the condition is significant, making it imperative to develop effective interventions for these individuals. Additionally, since many individuals with 22q11DS function in the borderline to low normal range of cognitive functioning, even mild cognitive gains could potentially result in a substantive improvement in functional outcomes and productivity in society.

We present data from the first study to employ a novel cognitive remediation program designed to improve cognitive deficits in adolescents with 22q11DS. The intervention consisted of a 12-week self-administered computerized cognitive remediation program (CCRP) based on the recognition that improvements in basic processes such as attention and memory lead to gains in overall cognition by enhancing brain plastic changes across multiple cortical areas (Fisher, Holland, Subramaniam, \& Vinogradov, 2009; Mahncke et al., 2006). The program was designed using exercises developed for the BrainWorks ${ }^{\mathrm{TM}}$ program by Posit Science. Because visual-spatial processing is a particular area of weakness for children with 22q11DS, visual as well as auditory exercises were chosen. The exercises were also selected to appeal to children and were piloted initially with four participants with 22q11DS to ensure that they were adapted to suit the participants' developmental and cognitive levels.

Our data indicate that this intervention is feasible, acceptable, and associated with good compliance and participant satisfaction. All participants were able to use the CCRP without 
help from their parents, and they had no difficulty in comprehending the instructions or completing the exercises. The majority completed the program. Lack of adherence was attributed in two of four cases to lack of computer access and in two cases to lack of motivation on the part of the child or her parents. The other participants completed the exercises despite the demands of homework, sports, and music lessons. Developmental delays in children with 22q11DS often make schoolwork and homework unusually challenging, a factor to be considered in implementing this type of intervention on a larger scale.

Most parents (71\%) reported that they were very or somewhat satisfied with their children's participation, and $82 \%$ reported that they would be willing to do the program again. Only $12 \%$ of children reported that they were upset at having to use the program. Most parents (71\%) reported that their children complained sometimes or often about working on the program, and 59\% reported that their children experienced frustration with the program. They attributed this to the program "freezing," an issue that the investigative team reported to Posit Science and which was resolved before the intervention ended. Most parents (88\%) predicted that their children would have been more likely to complete all the exercises if the 40-minute sessions had been shorter or fewer in number. Our adherence data indicate that participants in our study spent an average of 29 hours playing the exercises, close to the predicted 32 hours. This adherence rate was similar to or higher than those in other pilot studies of cognitive remediation in adults with schizophrenia (Fisher, Holland, Merzenich, \& Vinogradov, 2009) and in cognitive remediation studies involving children (Hardy, Willard, Allen, \& Bonner, 2012). Overall, we found that such a program is feasible for children with 22q11DS despite multiple academic and social problems that make adherence to an ancillary program potentially difficult.

The CCRP targeted specific areas of weakness in 22q11DS, including processing speed, working memory, planning, and problem solving, in addition to the domains of attention and memory. Our study was not powered to detect significant efficacy results. However, preliminary efficacy results showed medium to large effect sizes in the executive function domain, specifically in processing speed (Stroop Color-Word Times), and changes in the right direction in most targeted cognitive areas. We saw a larger effect size with the overall cognitive index change from T1 to T2 than with the individual cognitive domains in the intervention group, likely due to the aggregate effect of all the improvements across all the domains. We also saw a medium effect size associated with relative maintenance of GAF scores in the intervention group relative to the control group, although it would be premature to infer that the CCRP has an effect on global function given the short duration of the intervention. The improvements in the number of CPT hits may be due to practice effects, since we saw similar improvements in both the intervention and control groups, even though the CPT is said to be relatively robust to practice effects (Chen, Hsiao, Hsiao, \& Hwu, 1998). Overall, our results of improvements in cognition are encouraging. As with studies in schizophrenia, it is possible that more hours of training would be associated with more gains. This may be especially true for areas of particular weakness for children with 22q11DS, such as visual-spatial processing, where we saw small effect sizes for improvement (Spatial Span Backwards).

The limitations of the study include the small sample size and limited direct observation of participants. The small sample size also limited the number of treatment confounds that we could explore, although we are aware of moderators that could have affected our findings (e.g., language impairment, severity of psychopathology, and medication status). Since our goal was to demonstrate feasibility, the small sample size does not diminish the importance of our observations. Despite our observing participants playing games at only two time points, those observations, ongoing communication with the parents, variable week-to-week 
compliance, and responses on the satisfaction questionnaires enabled us to conclude that the children were playing the games themselves rather than the parents playing games for the children.

Our program has many advantages. First, it is a neuroplasticity-based intervention, developed based on the premise that improvement in the building blocks of cognition, such as attention and memory, can improve overall cognition (Adcock et al., 2009). The significant improvements in processing speed and improvements in the right direction in the other domains targeted are expected to result in changes in brain function, although this remains to be demonstrated. Second, we customized the exercises to specifically focus on both visual and auditory processes, with the former being a particular area of weakness in children with 22q11DS. Third, since children with 22q11DS have high rates of schizophrenia which is associated with decline in cognition, choosing a program that has been efficacious in adults with schizophrenia could provide us with an intervention that in the future may be effective in improving cognition and functionality in individuals with 22q11DS with and without psychotic illnesses.

In conclusion, we have demonstrated that a novel computerized cognitive remediation program is feasible and accepted by adolescents with 22q11DS and their families. Cognitive improvements were detected over the course of this program in targeted outcomes, and we anticipate that programs with larger sample sizes or longer duration would achieve stronger gains in cognition. Future topics to be examined in a randomized controlled trial would include potential moderators of treatment effects, generalization of results to academic performance and overall functioning, and durability of results beyond the intervention period.

\section{Acknowledgments}

The study was funded by R34-MH091314 (National Institutes of Mental Health) and a NARSAD Independent Investigator Award to the PI, Shashi, Vandana.

The authors are grateful to Posit Science for providing the computerized cognitive software used in the study.

\section{Bibliography}

Adcock RA, Dale C, Fisher M, Aldebot S, Genevsky A, Simpson GV, Vinogradov S. When top-down meets bottom-up: auditory training enhances verbal memory in schizophrenia. Schizophrenia Bulletin. 2009; 35(6):1132-1141. doi: 10.1093/schbul/sbp068. [PubMed: 19745022]

Baker KD, Skuse DH. Adolescents and young adults with 22q11 deletion syndrome: psychopathology in an at-risk group. British Journal of Psychiatry. 2005; 186:115-120. [PubMed: 15684233]

Biederman J, Faraone SV, Doyle A, Lehman BK, Kraus I, Perrin J, Tsuang MT. Convergence of the Child Behavior Checklist with structured interview-based psychiatric diagnoses of ADHD children with and without comorbidity. Journal of Child Psychology and Psychiatry. 1993; 34(7):1241-1251. doi: 10.1111/j.1469-7610.1993.tb01785.x. [PubMed: 8245144]

Chen WJ, Hsiao CK, Hsiao LL, Hwu HG. Performance of the Continuous Performance Test among community samples. Schizophrenia Bulletin. 1998; 24(1):163-174. [PubMed: 9502554]

Cornblatt BA, Risch NJ, Faris G, Friedman D, Erlenmeyer-Kimling L. The Continuous Performance Test, identical pairs version (CPT-IP): I. New findings about sustained attention in normal families. Psychiatry Research. 1988; 26(2):223-238. [PubMed: 3237915]

Curtiss K, Hooper S, Schoch K, Shashi V. Academic profile of children with 22q11.2 deletion syndrome: a case-control study delineating specific mathematics and reading disabilities. 2013 submitted. 
De Smedt B, Devriendt K, Fryns JP, Vogels A, Gewillig M, Swillen A. Intellectual abilities in a large sample of children with Velo-Cardio-Facial Syndrome: an update. Journal of Intellectual Disability Research. 2007; 51(Pt 9):666-670. doi: 10.1111/j.1365-2788.2007.00955.x. [PubMed: 17845235]

Debbane M, Glaser B, David MK, Feinstein C, Eliez S. Psychotic symptoms in children and adolescents with 22q11.2 deletion syndrome: Neuropsychological and behavioral implications. Schizophrenia Research. 2006; 84(2-3):187-193. [PubMed: 16545541]

Debbane M, Glaser B, Eliez S. Encoding and retrieval processes in velo-cardio-facial syndrome (VCFS). Neuropsychology. 2008; 22(2):226-234. doi: 10.1037/0894-4105.22.2.226. [PubMed: 18331165]

Delis, DC.; Kramer, JH.; Kaplan, E.; Ober, BA. California Verbal Learning Test-Children's Version (CVLT-C). The Psychological Corporation; San Antonio, TX: 1993.

Dempster AP, L. M. Rubin DB. Maximum likelihood from incomplete data via the EM algorithm. Journal of the Royal Statistical Society. Series B (Methodological). 1977; 39(1):1-38.

Driscoll DA, Budarf ML, Emanuel BS. A genetic etiology for DiGeorge syndrome: Consistent deletions and microdeletions of 22q11. American Journal of Human Genetics. 1992; 50(5):924933. [PubMed: 1349199]

Eack SM, Hogarty GE, Cho RY, Prasad KM, Greenwald DP, Hogarty SS, Keshavan MS. Neuroprotective effects of cognitive enhancement therapy against gray matter loss in early schizophrenia: Results from a 2-year randomized controlled trial. Archives of General Psychiatry. 2010; 67(7):674-682. doi: 10.1001/archgenpsychiatry.2010.63. [PubMed: 20439824]

Fisher M, Holland C, Merzenich MM, Vinogradov S. Using neuroplasticity-based auditory training to improve verbal memory in schizophrenia. American Journal of Psychiatry. 2009; 166(7):805-811. doi: 10.1176/appi.ajp.2009.08050757. [PubMed: 19448187]

Fisher M, Holland C, Subramaniam K, Vinogradov S. Neuroplasticity-based cognitive training in schizophrenia: An interim report on the effects 6 months later. Schizophrenia Bulletin. 2009 doi: $10.1093 / \mathrm{schbul} / \mathrm{sbn} 170$.

Hamer RM, Simpson PM. Last observation carried forward versus mixed models in the analysis of psychiatric clinical trials. American Journal of Psychiatry. 2009; 166(6):639-641. doi: 10.1176/ appi.ajp.2009.09040458. [PubMed: 19487398]

Hammond JL, Hirt M, Hall SS. Effects of computerized match-to-sample training on emergent fraction-decimal relations in individuals with fragile X syndrome. Res Dev Disabil. 2012; 33(1):111. doi: 10.1016/j.ridd.2011.08.021. [PubMed: 22093642]

Hardy KK, Willard VW, Allen TM, Bonner MJ. Working memory training in survivors of pediatric cancer: a randomized pilot study. Psycho-Oncology. 2012 doi: 10.1002/pon.3222.

Holmes J, Gathercole SE, Place M, Dunning DL, Hilton KA, Elliott JG. Working memory deficits can be overcome: Impacts of training and medication on working memory in children with ADHD. Applied Cognitive Psychology. 2010; 24(6):827-836. doi: 10.1002/acp.1589.

Hooker CI, Bruce L, Fisher M, Verosky SC, Miyakawa A, Vinogradov S. Neural activity during emotion recognition after combined cognitive plus social cognitive training in schizophrenia. Schizophrenia Research. 2012; 139(1-3):53-59. doi: 10.1016/j.schres.2012.05.009. [PubMed: 22695257]

Jolin EM, Weller RA, Weller EB. Psychosis in children with velocardiofacial syndrome (22q11.2 deletion syndrome). Current Psychiatry Reports. 2009; 11(2):99-105. [PubMed: 19302762]

Krikorian R, Bartok JA. Developmental data for the Porteus Maze Test. Clinical Neuropsychologist. 1998; 12(3):305-310. doi: 10.1076/clin.12.3.305.1984.

Lewandowski KE, Shashi V, Berry PM, Kwapil TR. Schizophrenic-like neurocognitive deficits in children and adolescents with 22q11 deletion syndrome. American Journal of Medical Genetics Part B: Neuropsychiatric Genetics. 2007; 144(1):27-36.

Lohaugen GC, Antonsen I, Haberg A, Gramstad A, Vik T, Brubakk AM, Skranes J. Computerized working memory training improves function in adolescents born at extremely low birth weight. Journal of Pediatrics. 2011; 158(4):555-561. e554. doi: 10.1016/j.jpeds.2010.09.060. [PubMed: 21130467] 
Mahncke HW, Bronstone A, Merzenich MM. Brain plasticity and functional losses in the aged: scientific bases for a novel intervention. Progress in Brain Research. 2006; 157:81-109. doi: 10.1016/S0079-6123(06)57006-2. [PubMed: 17046669]

McCloskey, G.; Maerlender, A. The WISC-IV integrated. In: Prifitera, A.; Saklofske, DH.; Weiss, LG., editors. WISC-IV clinical use and interpretation: Scientist-practitioner perspectives. Elsevier Academic Press; San Diego, CA US: 2005. p. 101-149.

Moss EM, Batshaw ML, Solot CB, Gerdes M, Donald-McGinn DM, Driscoll DA, Wang PP. Psychoeducational profile of the 22q11.2 microdeletion: A complex pattern. Journal of Pediatrics. 1999; 134(2):193-198. [PubMed: 9931529]

Murphy KC, Jones LA, Owen MJ. High rates of schizophrenia in adults with velo-cardio-facial syndrome. Archives of General Psychiatry. 1999; 56(10):940-945. [PubMed: 10530637]

Niklasson L, Rasmussen P, Oskarsdottir S, Gillberg C. Attention deficits in children with 22q.11 deletion syndrome. Developmental Medicine and Child Neurology. 2005; 47(12):803-807. doi: 10.1017/S0012162205001702. [PubMed: 16288669]

NIMH-CDISC. Computerized Diagnostic Interview Schedule for Children. 2004.

Papolos DF, Faedda GL, Veit S, Goldberg R, Morrow B, Kucherlapati R, Shprintzen RJ. Bipolar spectrum disorders in patients diagnosed with velocardio-facial syndrome: does a hemizygous deletion of chromosome 22q11 result in bipolar affective disorder? American Journal of Psychiatry. 1996; 153(12):1541-1547. [PubMed: 8942449]

Philip N, Bassett A. Cognitive, behavioural and psychiatric phenotype in 22q11.2 deletion syndrome. [Review]. Behavior Genetics. 2011; 41(3):403-412. doi: 10.1007/s10519-011-9468-z. [PubMed: 21573985]

Pulver AE, Nestadt G, Goldberg R, Shprintzen RJ, Lamacz M, Wolyniec PS, Housman D. Psychotic illness in patients diagnosed with velocardio-facial syndrome and their relatives. Journal of Nervous and Mental Disease. 1994

Shashi V, Keshavan M, Kaczorowski J, Schoch K, Lewandowski KE, McConkie-Rosell A, Kwapil TR. Socioeconomic status and psychological function in children with chromosome 22q11.2 deletion syndrome: implications for genetic counseling. Journal of Genetic Counseling. 2010; 19(5):535-544. doi: 10.1007/s10897-010-9309-x. [PubMed: 20680421]

Shprintzen RJ. Velo-cardio-facial syndrome: 30 Years of study. Developmental Disabilities Research Reviews. 2008; 14(1):3-10. doi: 10.1002/ddrr.2. [PubMed: 18636631]

Shprintzen RJ, Goldberg R, Golding-Kushner KJ, Marion RW. Late-onset psychosis in the velocardio-facial syndrome. American Journal of Medical Genetics. 1992; 42(1):141-142. [PubMed: 1308357]

Smith GE, Housen P, Yaffe K, Ruff R, Kennison RF, Mahncke HW, Zelinski EM. A cognitive training program based on principles of brain plasticity: Results from the Improvement in Memory with Plasticity-based Adaptive Cognitive Training (IMPACT) Study. Journal of the American Geriatric Society. 2009 doi: 10.1111/j.1532-5415.2008.02167.x.

Soderqvist S, Nutley SB, Ottersen J, Grill KM, Klingberg T. Computerized training of non-verbal reasoning and working memory in children with intellectual disability. Frontiers in Human Neuroscience. 2012; 6:271. doi: 10.3389/fnhum.2012.00271. [PubMed: 23060775]

Sonuga-Barke EJ, Brandeis D, Cortese S, Daley D, Ferrin M, Holtmann M, Sergeant J. Nonpharmacological interventions for ADHD: systematic review and meta-analyses of randomized controlled trials of dietary and psychological treatments. American Journal of Psychiatry. 2013; 170(3):275-289. doi: 10.1176/appi.ajp.2012.12070991. [PubMed: 23360949]

Stoddard J, Niendam T, Hendren R, Carter C, Simon TJ. Attenuated positive symptoms of psychosis in adolescents with chromosome 22q11.2 deletion syndrome. Schizophrenia Research. 2010; 118(13):118-121. doi: 10.1016/j.schres.2009.12.011. [PubMed: 20056393]

Stroop JR. Studies of interference in serial verbal reactions. Journal of Experimental Psychology. 1935; 18(6):643-662. doi: 10.1037/h0054651.

Wechsler, D. Intelligence Scale for Children-4th Edition. The Psychological Corporation; San Antonio, TX: 2003.

Wechsler, D. Wechsler Adult Intelligence Scale-. 4th Edition. The Psychological Corporation; San Antonio, Texas: 2008. 
Woodin M, Wang PP, Aleman D, Donald-McGinn D, Zackai E, Moss E. Neuropsychological profile of children and adolescents with the 22q11.2 microdeletion. Genetics in Medicine. 2001; 3(1):3439. [PubMed: 11339375]

Wykes T, Huddy V, Cellard C, McGurk SR, Czobor P. A meta-analysis of cognitive remediation for schizophrenia: methodology and effect sizes. American Journal of Psychiatry. 2011; 168(5):472485. doi: 10.1176/appi.ajp.2010.10060855. [PubMed: 21406461]

Young AS, Shashi V, Schoch K, Kwapil T, Hooper SR. Discordance in diagnoses and treatment of psychiatric disorders in children and adolescents with 22q11.2 Deletion Syndrome. Asian Journal of Psychiatry. 2011; 4(2):119-124. doi: 10.1016/j.ajp.2011.03.002. [PubMed: 21743818] 


\section{Highlights}

We piloted a novel cognitive remediation program for children with 22q11DS

Open-label design with 13 children with 22q11DS in intervention and 10 in control group

Preliminary analyses show that the program is feasible and acceptable to subjects Significant improvements in neurocognition with medium to large effect sizes 
Table 1

Demographic table

\begin{tabular}{|l|l|l|l|}
\hline Demographic measure & Intervention group mean (SD), range & Control group mean (SD), range & p-value \\
\hline Age & 14.75 years (1.41), 12-17 & 14.99 years (2.35), 12-17 & 0.563 \\
\hline Gender & $46 \%$ male, 54\% female & $60 \%$ male, 40\% female & 0.585 \\
\hline Race & $85 \%$ Caucasian, 15\% African-American or biracial & $100 \%$ Caucasian & 0.118 \\
\hline FSIQ ${ }^{*}$ & $79.8(15.4), 59-109$ & $76.8(14.3), 62-106$ & 0.641 \\
\hline SES ${ }^{* *}$ & $28.77(11.66), 11-58$ & $33.78(18.42), 11-61$ & 0.484 \\
\hline GAF & $62.33(11.61), 52-81$ & $64.0(8.4), 41-81$ & 0.789 \\
\hline
\end{tabular}

* estimated using Matrix Reasoning and Vocabulary subtests of the WISC-IV

**

rated using Hollingshead's two-factor index of parental education and employment 
Table 2

Parent surveys regarding CCRP.

\begin{tabular}{|l|l|l|}
\hline Parent surveys, CCRP (n= 17) & Answers \\
\hline Question & Never/Rarely: 29\% & Sometimes/Often: 71\% \\
\hline $\begin{array}{l}\text { How often did your child complain about having to complete exercises on the } \\
\text { computer? }\end{array}$ & Never/Rarely: 76\% & Sometimes/Often: 24\% \\
\hline $\begin{array}{l}\text { How often did YOU feel like complaining about your child having to complete } \\
\text { exercises on the computer? }\end{array}$ & Never/Rarely: 100\% & Sometimes/Often: 0 \\
\hline How often did you help your child with the exercises? & Never/Rarely: 41\% & Sometimes/Often: 59\% \\
\hline $\begin{array}{l}\text { How often did your child experience frustration while using the computer } \\
\text { program? }\end{array}$ & No: 50\% & Yes: 50\% \\
\hline $\begin{array}{l}\text { Do you think your child would have completed the exercises even without the } \\
\text { opportunity to gain a payment? }\end{array}$ & More money: 12\% & Fewer/shorter sessions: 88\% \\
\hline $\begin{array}{l}\text { Which of the following might make your child more likely to complete all the } \\
\text { exercises? }\end{array}$ & Very/Somewhat: 71\% & Neutral/Dissatisfied: 29\% \\
\hline How satisfied were you with your child's participation in the study? & Definitely/I think so: 82\% & No, I don't think so: $18 \%$ \\
\hline Would you be willing to do this program again? &
\end{tabular}

The 17 surveys represented in the table are a combination of the 6- and 12-week surveys, with 8/9 surveys received at the 6-week time point and $9 / 9$ at the end of 12 weeks (Intervention group $n=13$ ). At both time points the four participants that did not complete all the exercises were nonrespondents. 
Table 3

Responses from participants with 22q11DS who were in the intervention group.

\begin{tabular}{|l|l|l|l|}
\hline Participant surveys, CCRP (n= 17) \\
\hline Question & Answers & Just right: $29 \%$ & Too hard: $6 \%$ \\
\hline $\begin{array}{l}\text { Do you think the questions on the computer exercise } \\
\text { were: }\end{array}$ & Easy: $65 \%$ & Didn't really care: $47 \%$ & Upset: $12 \%$ \\
\hline $\begin{array}{l}\text { When it was time to use the computer program, did you } \\
\text { feel: }\end{array}$ & Excited: $41 \%$ & Hard to stay focused: $23 \%$ \\
\hline When you were using the program, was it: & Easy to stay focused: $18 \%$ & Neutral: $59 \%$ & Just happy it's over!: $56 \%$ \\
\hline Since you've completed the program, do you feel: & Proud of myself: $25 \%$ & The same: $19 \%$ & \\
\hline
\end{tabular}

Surveys include those obtained at $6(n=8 / 9)$ and 12 weeks $(n=9 / 9)$ from the interventions participants that completed the program 


\section{Table 4}

Comparisons of cognitive outcome variables for the intervention $(n=13)$ versus control $(n=10)$ groups at pre-test and post-test.

\begin{tabular}{|c|c|c|c|c|c|c|c|c|c|}
\hline Domain/Task & $\begin{array}{c}\text { Intervention } \\
\text { pre-test } \\
\text { mean (SD) }\end{array}$ & $\begin{array}{c}\text { Intervention } \\
\text { post-test } \\
\text { mean (SD) }\end{array}$ & Effect size & $\begin{array}{l}\text { Control pre- } \\
\text { test mean } \\
\text { (SD) }\end{array}$ & $\begin{array}{c}\text { Control } \\
\text { post-test } \\
\text { mean }(\mathrm{SD})\end{array}$ & Effect size & t-value & p-value & Between-group effect size \\
\hline \multicolumn{10}{|c|}{ Attention Domain } \\
\hline CPT Hits & $7.62(1.74)$ & $17.52(6.85)$ & 4.80 & $9.39(1.90)$ & $18.69(5.35)$ & 4.51 & 0.20 & .844 & .29 \\
\hline CBCL Attention & $61.83(9.23)$ & $60.00(7.53)$ & -.22 & $60.42(7.55)$ & $61.70(8.68)$ & .15 & -1.14 & .276 & -.37 \\
\hline \multicolumn{10}{|c|}{ Memory Domain } \\
\hline CVLT Total & $39.81(14.40)$ & $42.06(12.58)$ & .17 & $41.20(11.45)$ & $39.63(10.13)$ & -.12 & .85 & .409 & .30 \\
\hline CVLT Short-Delay Free & $-.72(1.05)$ & $-.39(.94)$ & .36 & $-.17(.67)$ & $-.73(1.42)$ & -.60 & 1.62 & .125 & .97 \\
\hline $\begin{array}{l}\text { CVLT Long Delay } \\
\text { Cued }\end{array}$ & $-.34(1.21)$ & $.69(1.23)$ & -.34 & $-.01(.67)$ & $-.09(.84)$ & -.08 & -.51 & .615 & -.26 \\
\hline \multicolumn{10}{|c|}{ Executive Function Domain } \\
\hline Mazes Passed & $9.69(2.16)$ & $10.70(2.26)$ & .48 & $11.44(1.65)$ & $11.15(1.60)$ & -.14 & 1.02 & .325 & .61 \\
\hline Mazes Credits & $12.94(2.84)$ & $11.81(3.65)$ & -.41 & $13.65(2.52)$ & $11.52(3.50)$ & -.78 & .74 & .472 & .36 \\
\hline Digits Backwards & $7.24(2.95)$ & $7.78(2.87)$ & .21 & $8.06(2.11)$ & $8.35(2.22)$ & .11 & .25 & .804 & .09 \\
\hline Spatial Span Backwards & $6.77(3.83)$ & $7.34(3.48)$ & .16 & $7.70(3.16)$ & $7.87(2.69)$ & .05 & .27 & .793 & .11 \\
\hline Stroop Time 1 & $14.73(4.33)$ & $12.26(3.43)$ & -.56 & $12.25(4.43)$ & $13.12(5.30)$ & .20 & -2.19 & $.044^{*}$ & -.76 \\
\hline Stroop Time 2 & $38.30(6.70)$ & $30.26(6.63)$ & -.75 & $38.52(14.95)$ & $34.87(14.41)$ & -.34 & -.92 & .373 & -.41 \\
\hline \multicolumn{10}{|c|}{ Cognitive Composite } \\
\hline Composite & $-.35(.93)$ & $.98(1.07)$ & 1.33 & $.44(.93)$ & $.98(1.20)$ & .54 & 2.62 & $.019^{*}$ & .79 \\
\hline
\end{tabular}

All analyses were adjusted for age and gender effects, as well as differences in baseline cognition between the intervention and control groups.

Medium and large effect sizes are in bold.

* $<<.05$ 